\title{
The effect of a high fat or high carbohydrate diet on the immune system of young Balb/c mice
}

\author{
B. E. Martínez-Carrillo ${ }^{1,2}$, R. A. Jarillo-Luna ${ }^{2}$, V. Rivera-Aguilar ${ }^{2}$, R. Campos-Rodríguez ${ }^{2}$, \\ R. V. Pardo-Morales ${ }^{1}$, D. Jiménez-López ${ }^{1}$ and R. Valdés-Ramos ${ }^{1}$ \\ ${ }^{1}$ Center for Research and Graduate Studies in Health Sciences, Faculty of Medicine, Universidad Autónoma del Estado \\ de México and ${ }^{2}$ Instituto Politécnico Nacional, Laboratorio de Inmunología
}

Inappropriate lifestyles have shifted dietary patterns from natural to processed foods as well as to high fat, high simple carbohydrates and low fibre diets ${ }^{(1)}$. High fat or high carbohydrate diets have different metabolic effects and may cause diseases such as diabetes, atherosclerosis or obesity ${ }^{(2)}$. These effects have also been observed on some areas of the immune system; however, little is known about changes caused by diet on the gut mucosal immunity, which is the first point of entry for dietary components. The objective of this study was to evaluate the effect of a high fat (HFD) or a high carbohydrate (HCD) diet on T-cells from lamina propria in a murine model. We used three groups $(n=8)$ of 21-day-old Balb/c mice; Control, HFD, HCD (Table 1); small intestine (Peyer's patches were eliminated) samples were obtained after 9 weeks. After purification of T-cells, lymphocytes were cultivated with phytohaemagglutinin (PHE) for $48 \mathrm{~h}$, supernatants were collected for determination of IL-2, IL-4, IL-5, TNF- $\alpha$ and IFN- $\gamma$, and T-cell populations using anti-CD3 + , CD4 + and CD8 + were analysed by flow cytometry. HFD mice had lower T-CD3 + $(30.92 \pm 3.62 \%)$, T-CD4 + $(1.48 \pm 0.33 \%)$ and T-CD8 + $(3.46 \pm 1.87 \%)$, while HCD mice had increased values of all T-CD3+ $(56.36 \pm 6.76 \%)$, T-CD $4+(20.24 \pm 4.5 \%)$ and $\mathrm{T}-\mathrm{CD} 8+$ $(26.93 \pm 4.67 \%)$ when compared to Controls $(44.34 \pm 3.84 \%, 19.98 \pm 3.59 \%$ and $22.29 \pm 3.82 \%$, respectively) (Fig. 1). Data were statistically different by ANOVA $(F=83.38, P<0.0001)$. Cytokine secretion was found increased in both HCD (IL-2 5.26 \pm 1.68 pg/ml, IL-5 $16.65 \pm 2.02 \mathrm{pg} / \mathrm{ml}$ and TNF- $\alpha 14.52 \pm 2.35 \mathrm{pg} / \mathrm{ml}$ ) and HFD (IL-2 $4.60 \pm 0.930 \mathrm{pg} / \mathrm{ml}, \quad$ IL-5 $10.78 \pm 2.32 \mathrm{pg} / \mathrm{ml}, \mathrm{TNF}-\alpha$ $18.13 \pm 2.05 \mathrm{pg} / \mathrm{ml}$; compared to controls $(3.03 \pm 0.568,8.27 \pm 1.25$ and $9.28 \pm 1.88$, respectively). HFD is able to affect more drastically the mucosal immune system with a decrease in all T-cell populations and a higher TNF- $\alpha$ secretion. HCD on the other hand, increases T lymphocytes as well as IL-2 and IL-5. We can conclude that a high-fat diet has a more deleterious effect on the immune system in mice. This project was financed by Universidad Autónoma del Estado de México.

Table 1. Content in percentage of nutrient contribution for each experimental diet

\begin{tabular}{llcrr}
\hline & & Control & HCD & HFD \\
\hline Carbohydrate & Starch & 39.74 & 0.00 & 0.00 \\
& Maltodextrin & 13.20 & 33.17 & 20.13 \\
& Sucrose & 10 & 33.18 & 20.13 \\
Total & & $\mathbf{6 2 . 9 4}$ & $\mathbf{6 6 . 3 5}$ & $\mathbf{4 0 . 2 6}$ \\
Fat & Oil of soybean & 7.01 & 2.37 & 2.91 \\
& Lard & 0.00 & 1.93 & 20.69 \\
Total & & $\mathbf{7 . 0 1}$ & $\mathbf{4 . 3 0}$ & $\mathbf{2 3 . 6 0}$ \\
Protein & Casein & 20 & 18.95 & 23.30 \\
Total & & $\mathbf{8 9 . 9 6}$ & $\mathbf{8 9 . 6 0}$ & $\mathbf{8 7 . 1 6}$ \\
\hline
\end{tabular}

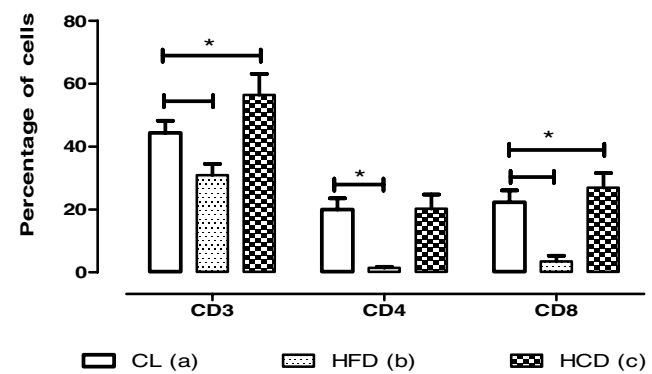

Figure 1. Percentage of T-cells in lamina propia of small intestine of Balb/c mice fed with three diets. The values represent the means \pm sD of three determinations, each group with $n=8$, ANOVA, $P<0.0001$. *Post hoc Tukey test comparing the three groups (a, b and c) showed differences between groups, with an IC of $95 \%, P<0.0001 ; \mathrm{CD}$ (cluster of differentiation 3, 4 and 8); (a) CL (control), (b) HFD (high fat diet) and (c) HCD (high carbohydrate diet).

1. Lunn J \&Theobald H (2007) Br Nutr Found 31, 178-224.

2. Fritsche K (2007) Lipids 42, 961-979. 